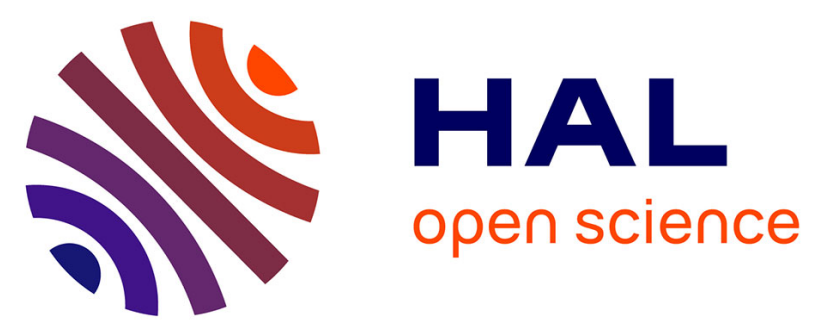

\title{
Automatic measurement of the sinus of Valsalva by image analysis
}

Fabrice Mairesse, Cédric Blanchard, Arnaud Boucher, Tadeusz Sliwa, Alain

Lalande, Yvon Voisin

\section{- To cite this version:}

Fabrice Mairesse, Cédric Blanchard, Arnaud Boucher, Tadeusz Sliwa, Alain Lalande, et al.. Automatic measurement of the sinus of Valsalva by image analysis. Computer Methods and Programs in Biomedicine, 2017, 148, pp.123-135. 10.1016/j.cmpb.2017.06.014 . hal-01577247

\section{HAL Id: hal-01577247 \\ https://u-bourgogne.hal.science/hal-01577247}

Submitted on 6 Jul 2021

HAL is a multi-disciplinary open access archive for the deposit and dissemination of scientific research documents, whether they are published or not. The documents may come from teaching and research institutions in France or abroad, or from public or private research centers.
L'archive ouverte pluridisciplinaire HAL, est destinée au dépôt et à la diffusion de documents scientifiques de niveau recherche, publiés ou non, émanant des établissements d'enseignement et de recherche français ou étrangers, des laboratoires publics ou privés. 


\title{
Automatic measurement of the sinus of Valsalva by image analysis
}

\author{
Fabrice Mairesse $^{\mathrm{a} *}$, Cédric Blanchard ${ }^{\mathrm{a}}$, Arnaud Boucher ${ }^{\mathrm{a}}$, Tadeusz Sliwa ${ }^{\mathrm{a}}$, Alain Lalande ${ }^{\mathrm{b} f}$, \\ Yvon Voisin ${ }^{\mathrm{a}}$
}

\footnotetext{
a Le2i FRE2005, CNRS, Arts et Métiers, Univ. Bourgogne Franche-Comté, av. des Plaines de l'Yonne, BP16, 89010 Auxerre Cedex, France ${ }^{b}$ Le2i FRE2005, CNRS, Arts et Métiers, Univ. Bourgogne Franche-Comté, 9 av. A. Savary, BP 47870, 21078 Dijon Cedex, France ' Service de Spectroscopie-RMN, CHU Dijon, 14 rue Paul Gaffarel, BP 77908, 21079 Dijon Cedex, France
}

\begin{abstract}
Background and Objectives: Despite the importance of the morphology of the sinus of Valsalva in the behavior of heart valves and the proper irrigation of coronary arteries, the study of these sinuses from medical imaging is still limited to manual radii measurements. This paper aims to present an automatic method to measure the sinuses of Valsalva on medical images, more specifically on cine MRI and Xray CT. Methods: This paper introduces an enhanced method to automatically localize and extract each sinus of Valsalva edge and its relevant points. Compared to classical active contours, this new image approach enhances the edge extraction of the Sinus of Valsalva. Our process not only allows image segmentation but also a complex study of the considered region including morphological classification, metrological characterization, valve tracking and 2D modeling. Results: The method was successfully used on single or multiplane cine MRI and aortic CT angiographies. The localization is robust and the proposed edge extractor is more efficient than the state-of-the-art methods (average success rate for MRI examinations $=84 \% \pm 24 \%$, average success rate for CT examinations=89\% $\pm 11 \%$ ). Moreover, deduced measurements are close to manual ones. Conclusions: The software produces accurate measurements of the sinuses of Valsalva. The robustness and the reproducibility of results will help for a better understanding of sinus of Valsalva pathologies and constitutes a first step to the design of complex prostheses adapted to each patient.
\end{abstract}

\section{Introduction}

The sinus of Valsalva (SV) is a cavity at the base of the aortic root between the sinotubular junction and the cardiac valves [1-3] (Fig. 1). In a normal morphology, the SV is tricuspid: it is composed by three anatomic dilations called cusps (Fig. 2a). Each cusp is associated with a valve. The junction between two valves along the aortic wall is called a commissure and defines the limit of each cusp. The natural center of the SV is the location where valves touch each other during their closure in the diastolic phase. Between two commissures, the cusp point is determinated with farthest edge points from the center.

Bicuspid SV is the most common congenital cardiac abnormality and affects the morphological structure of the SV [4,5] (Fig. 2b). It can be congenital [6] or, more ordinary, due to the raphe of two anatomic leaflets. This leads to only two functional leaflets.

Another pathology that could affect SV whatever the number of cusps is an abnormal dilatation: if the maximum diameter of the

\footnotetext{
* Corresponding author.

E-mail address: fabrice.mairesse@u-bourgogne.fr (F. Mairesse).
}

aorta exceeds a well-defined threshold, an aortic root replacement can be considered [7]. However, aortic root prostheses are almost cylindrical. Hence all radii are almost equal. In this case, commissures are localized via the valve and each cusp point is the farthest edge point from the commissures between two of them.

Over the past decade, the aortic valve surgical procedures are on the increase [8]. When a replacement of the aortic root is required, the shape of the prosthesis must be perfectly adapted to the morphology of the patient as it is important for valve closure and stress distribution [9]. However nowadays the aortic root measurement on medical images is still subjective and not normalized $[10,11]$. Even if SV do not have a cylindrical structure, but are trifoliate, its measurement is often manually performed from a sagittal long axis view [12] which is not the most relevant orientation. Moreover, this manual method introduces inter and intra-observer variations.

In this paper, the study of the SV is extended to multiplane cine-MRI and static X-ray computed tomography in order to decrease the influence of the choice of plane and its movements. After a presentation of the main geometrical properties of the $\mathrm{SV}$, a new geometrical model is proposed. Therefore mathematical 


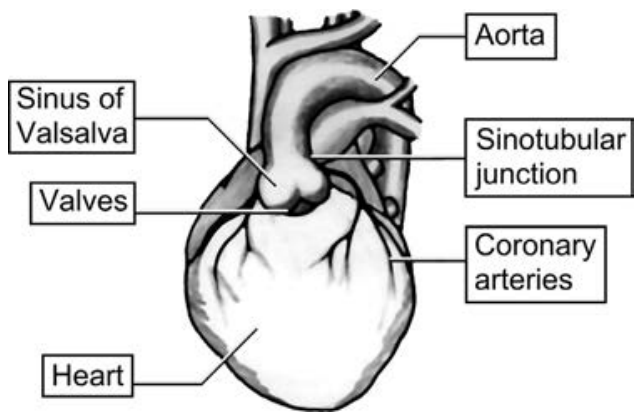

Fig. 1. The sinus of Valsalva and surrounding organs.

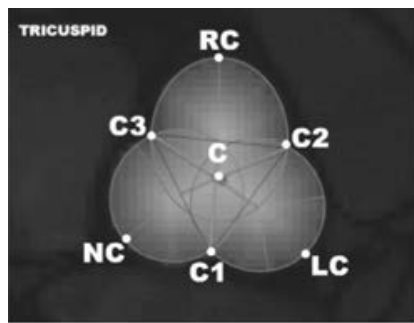

a)

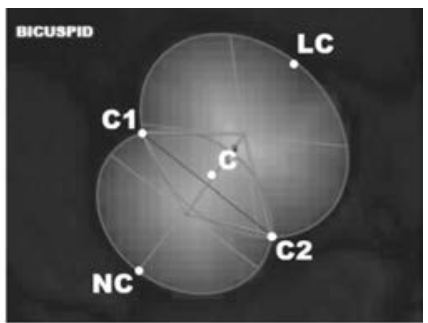

b)
Fig. 2. Location of relevant points and modeling of a tricuspid SV (a) and a bicuspid SV without the right coronary cusp(b) as an overlapping of ellipses. There are also two other cases for bicuspid SV depending on the missing cusp. $\mathrm{C}=$ center; C1,C2,C3: commissures; LC,RC,NC: Left Coronary Cusp, Right Coronary Cusp and Non Coronary Cusp.

morphology has been chosen as the most natural technique to apply on tomographic images of SV. The three main steps of processing are the localization of the SV, the extraction of its edge and the detection of relevant points. Particularly, a new morphological tool, the Aurora Transform (AT), is introduced and then used to improve the automatic extraction of SV edges compared to previous studies $[1,13]$. The efficiency and the reliability of each step are assessed and discussed.

The proposed methods allow image registration, 2D modeling, valve tracking, morphological classification and metrological characterization of SV. This work leads to a software dedicated for a quick and reliable analysis of the SV and the aortic root.

\section{Modality and data rendering}

In medical image analysis, the modality specifications and anatomic geometry knowledge are crucial. These aspects compose the a priori knowledge. They are the tools to compute data in intelligent way, to obtain a meaningful computer assisted analysis.

In this section, methods and conditions of acquisition that allow to correctly examine the aortic root and the SV are defined. According to the selected planes, the main geometrical properties of the shapes depicted by these SV are then described. Afterward, our contribution to the assisted localization, extraction and analysis of the SV is presented, including the design of pipelines composed by existing tools of image processing and the definition of a new useful one.

\subsection{Modality specifications}

The commonly employed techniques for aortic root examinations are echography, Magnetic Resonance Imaging (MRI) and X-ray Computed Tomography (CT). Even if a sagittal long axis view in transthoracic echography is often used $[12,14]$, there is no global consensus for the measurement of aortic root dimensions. Moreover, due to its complexity, the measurement of the SV shape is

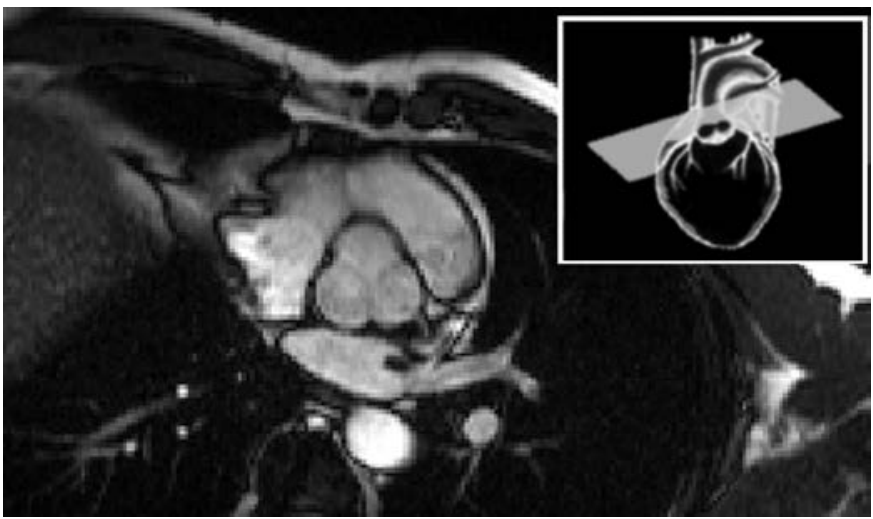

Fig. 3. The aortic valve cross-section plane. Example from a MR image.

not reliable in this plane. Tomographic methods, such as CT and MRI, allow to choose one or several acquisition planes and Burman and al. have already proposed to study the SV in an aortic valve cross-section plane [15] (Fig. 3), perpendicular to the two left ventricular outflow tract planes. The trifoliate shape of the SV can be studied from this plane, even if there is plane motion during cine-MRI acquisition [1]. With some planes parallel to the aortic valve crosssection plane and covering the whole aortic root, multiplane MRI and CT naturally decrease the effect of these unwanted movements on measurements and analysis of the SV.

\subsection{Anatomic properties and geometrical modeling}

\subsubsection{Anatomic presentation}

In an aortic valve cross-section plane, if the complete geometry and the size of a SV is not known in advance, some properties of the SV can be listed in order to enclose a priori information in our global processing:

1. On MR images acquired with Steady-State Free-Precession (SSFP) sequences and on CT images after injection of contrast agent, SV are quite bright regions;

2. They are star domains: there is a point inside the SV such that each segment between the SV edge and this point is included inside the SV;

3. Their size is in an interval of bounded values.

4. Their size in a slice is almost constant during a cardiac cycle.

5. During breath-hold, respiratory movements and myocardial displacement are reduced.

6. Considering multi-slices acquisitions, there is only a small variation of aortic root position and size between two successive slices. We call this property the spatial redundancy of the aortic root.

These properties are always true for all performed examinations, whatever the acquisition technique used.

\subsubsection{Mathematical rendering}

A geometrical framework must be defined for the various shapes of SV. In cross-sectional planes, the SV prosthesis and aortic root above the sinotubular junction can be model as a circle or an ellipse. Bicuspid and tricuspid SV can also be naturally seen as an overlapping of ellipses (Fig. 2).

An elliptical object is an object whose projection in the discrete image plane can be assimilated to a discrete ellipse. Let $a_{k}, k \in$ $\llbracket 1 ; n \rrbracket$ be the projection in the image plane of $n$ elliptical objects $(n>1)$ that constitute a simply connected shape (it consists of one piece and does not have any holes) $E=\bigcup_{k=1}^{n} a_{k}$. Let $\|$ be the cardinal number defining the number of pixels in a set and let $\nabla \ominus$ 
Table 1

Number of frames according to data types.

\begin{tabular}{llll}
\hline & Min & Max & Mean \\
\hline Cine-MRI (one plane) & 19 & 50 & 30 \\
Multi-planes cine-MRI & 45 & 224 & 80 \\
CT & 22 & 35 & 27 \\
\hline
\end{tabular}

be the morphological internal gradient [16] that provides the 8connected edge of a shape by keeping interior pixels which have an exterior pixel as neighbor.

Let $\Phi_{E}$ and $\Psi_{E}$ be two functions related to the structure of these shapes:

- $\Phi_{E}$ is the participation of each object projection in global shape edge:

$\forall k \in \llbracket 1 ; n \rrbracket, \Phi_{E}\left(a_{k}\right)=\frac{\left|\nabla^{\ominus}\left(a_{k}\right) \cap \nabla^{\ominus}(E)\right|}{\left|\nabla \ominus\left(a_{k}\right)\right|}$

$\Phi_{E}$ is between 0 and 1 . If there is only one object, its participation is $100 \%$, whereas if a region is entirely overlapped or surrounded by others, its participation is null.

- The regions superposition $\Psi_{E}$ can be quantified by following function:

$\forall k \in \llbracket 1 ; n \rrbracket, \Psi_{E}\left(a_{k}\right)=\frac{|E|-\left|E-a_{k}\right|}{|E|} \cdot \frac{\sum_{i=1}^{n}\left|a_{i}\right|}{\left|a_{k}\right|}$

The first term in the equation 2 is the area where only the considered object projection $\left(a_{k}\right)$ is present, normalized by the global area. The second term is a weighting linked with the size of the considered region compared with others. A high contribution means that a region is almost totally distinct from the others.

The complexity of a shape depends on the number of elliptical objects that define it and the choice of two intervals of tolerance for $\Phi_{E}$ and $\Psi_{E}$.

The shapes drawn by the SV in the image plane can be seen as a star domain and a subset of shapes composed by partially overlapped ellipses with the following constraints:

- The eccentricity $e$ must be in $[0 ; 0.4]$ to prevent unrealistic cusps.

- $\Phi_{E}$ and $\Psi_{E}$ must be chosen in the interval [0.25; 0.66];

- $k \leq 3$ and for $k=3, \forall(k) \in \llbracket 1 ; 3 \rrbracket, \nabla^{\ominus}\left(a_{k}\right) \cap \nabla^{\ominus}(E)$ is simply connected. In other words, the three ellipses make a cluster, not a sequence;

These limits have been empirically estimated on ground reality and the difference between the sizes of ellipses is also indirectly controlled by the limits of the superposition $\Psi_{E}$.

The position of commissures is directly given by the junctions of the edge of ellipses along the global edge, i.e. the extremities of each set of edge pixels $\{\operatorname{edg} e(E) \cap e d g e(a k)\}$.

\section{Data}

The data set is composed of 44 single plane cine-MRI, 8 multiplane cine-MRI and 4 Aortic CT angiographies.

A single plane cine-MRI has from 19 images to 50 images (30 images on average) whereas a multi-plane cine-MRI has from 45 images to 224 images (80 images on average) (Table 1). Regarding the multi-plane cine-MRI examinations, one of them has 7 planes, three have 5 planes and the four others have 3 planes. The resolution of the first mentioned examination is equal to $1.56 \mathrm{~mm}$ per pixel whereas the resolution of the others is equal to $0.96 \mathrm{~mm}$ per pixel.
The preferred file format is the DICOM format (Digital Imaging and Communications in Medicine) that contains unmodified images (Fig. 4a). However, in order to assess the robustness of our methods, one CT examination saved as DICOM and three CT examinations saved as JPEG images (high quality/low compression rate) were added to the data set. These four examinations contain embedded text around the SV (Fig. 4.b).

No temporal information is available, but there are many planes along the aortic root. The four CT examinations have respectively $22,23,28$ and 35 slices. The unavoidable radiation exposure is obviously the drawback of this technique, but it allows a good contrast for the aorta. Moreover CT provides a better spatial resolution than cine-MRI.

MRI was performed on a $1.5 \mathrm{~T}$ magnetic resonance whole body imager (Siemens Magnetom Avanto, Siemens Medical Solution, Germany) using a phased array thoracic coil. The cine-MR images were acquired using a breath-hold ECG-gated SSFP sequence covering the whole cardiac cycle with the following acquisition parameters:

- Repetition time (TR): $1.54 \mathrm{~ms}$;

- Echo time (TE): $1.49 \mathrm{~ms}$;

- 17 lines per segment;

- Pulse flip angle $(\alpha)$ : 65 degrees;

- Slice thickness: $5 \mathrm{~mm}$;

- Temporal resolution: $27 \mathrm{~ms}$ per image;

- Isotropic in plane spatial resolution: from $0.7 \mathrm{~mm}$ per pixel to $2.0 \mathrm{~mm}$ per pixel, according to the patient examination (mean $=1.2 \mathrm{~mm}$, standard deviation $=0.4 \mathrm{~mm}$ );

- Number of planes: between 1 and 7 .

Cine-MRI provides temporal information including the movement and the deformation of the SV and the opening and the closing of the valves. Even without contrast media, the contrast between the blood and tissues is high. On the other hand, temporal and spatial resolutions are limited by the duration of the breathhold. Hence, quality and quantity of images directly depends on patient capabilities.

Aortic CT angiography was performed using a secondgeneration dual-source 128-MDCT (Definition Flash, Siemens Medical Solutions, Erlangen, Germany), with ECG-triggering. The following parameters were used:

- $2 \times 64 \times 0.6 \mathrm{~mm}$ collimation;

- $0.28 \mathrm{~s}$ rotation time;

- pitch of 0.25 ;

- $120 \mathrm{kV}$ tube voltage;

- 320 mAs tube current-time product.

A contrast agent was injected intravenously at a rate of $5 \mathrm{~mL} / \mathrm{s}$ (80 mL Iomeron [400 mg iodine $/ \mathrm{mL}$ ], Bracco Altana Pharma, Milan, Italy). Axial cross-sectional image reconstructions of the aortic sinus were performed and analyzed on a separate workstation using a mediastinal window in order to select relevant intensities $(1 \mathrm{~mm}$ axial cross-sectional, perpendicular to the vessel center line, Window: 600-800, Level: 100-200).

\section{Methods}

The aim of this method is the segmentation and the measurement of SV. The proposed model consists of three parts. The first part is the preprocessing part for SV localization. The second part deals with SV segmentation and edge extraction. The last part aims to SV measurements.

The methods involved in the first part are as follows: as a first step, a normalization and binarization are done. In the second step, an organs segmentation is done using mathematical morphology 


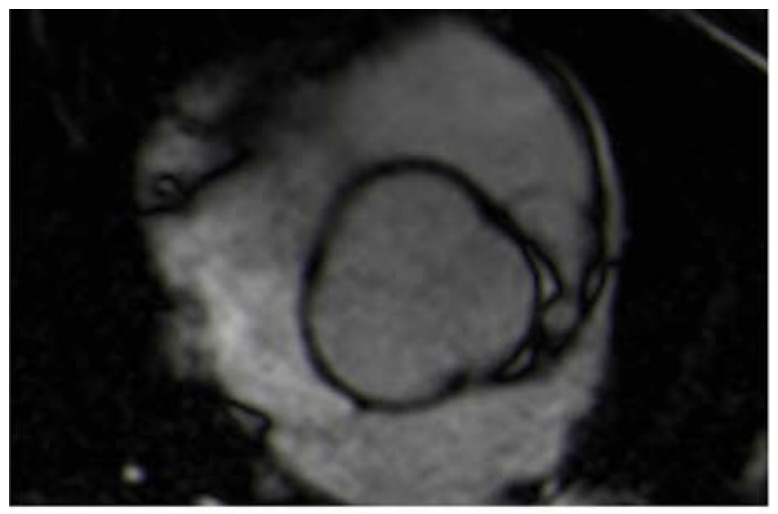

a)

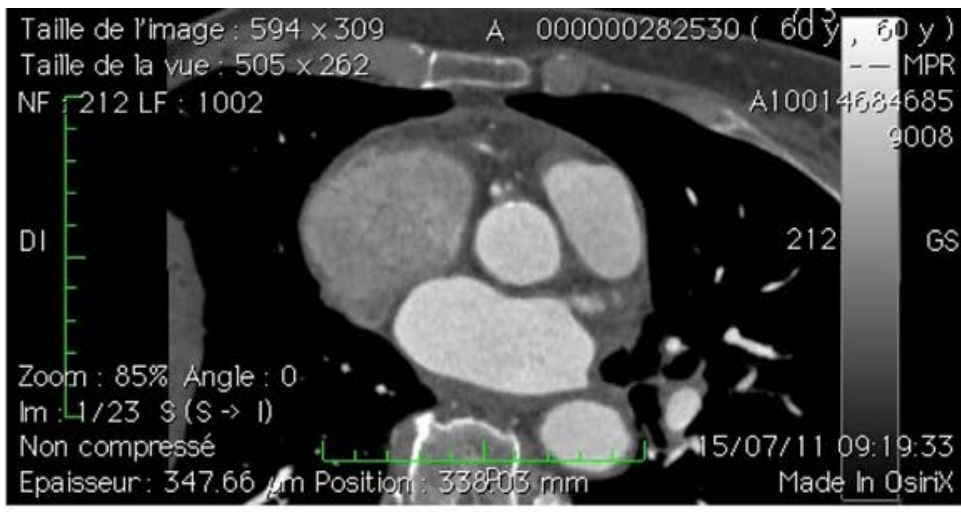

b)

Fig. 4. Database image examples. a) MRI image. b) CT image with embedded text.
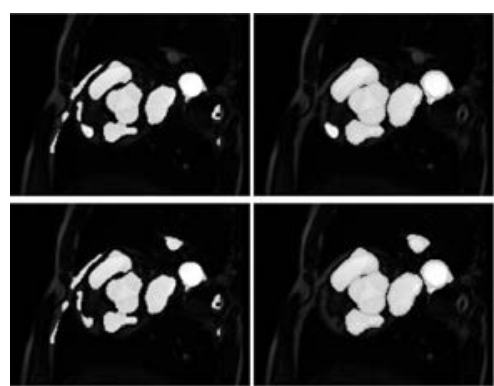

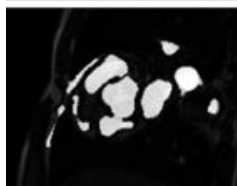

a)

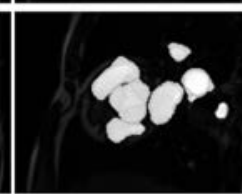

b)

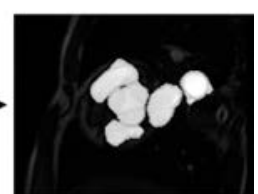

c)
Fig. 5. Main steps of the extraction of bright regions. Bright pixels (a) are found and bright regions (b) are identified in each image of the examination. Finally, a correction is applied (c) in order to only preserve the regions that are visible in the previous, current and next frames.

processing and a priori information. In the third step, blob trajectories are computed to identify aorta root (Fig.5). In the second part, a SV edges extraction method is implemented. This new polar geodesic operator named Aurora Transform (AT) allows us to precisely define SV edges despite connections with neighbor organs. The final part deals with the computer aided diagnosis process. To carry out this task, several processes are done: SV edge relevant points detection, temporal tracking and measurements.

In the following, a $3 \mathrm{D}+\mathrm{t}$ data set is considered. We note $I_{z}(k)$ as the frame number $k$ in the slice number $z$.

\subsection{SV localization}

Since the operators of mathematical morphology directly relate to shape, they are easy-to-use and efficient for image segmentation [17-19]. Since the body globally does not move during the examination, the area $A_{z}$ locating the interior of the body in each slice can be deduced from only the first image $I_{z}(1)$. Let $B_{R 1}$ be a disk whose radius is coherent with the common mean body size and $B_{R 2}$ be a disk with a radius close to the mean thickness of the fat around the body. $A_{z}$ is defined as followed:

$$
A_{z}=\gamma\left(\varphi\left(T_{c}\left(I_{z}(1)\right), B_{R 1}\right), B_{R 2}\right)
$$

where $\gamma$ is the morphological opening and $\varphi$ is the morphological closing. The morphological closing fills dark organs inside the body while the opening erases superficial fat signals.

\subsubsection{Organs extraction}

Considering acquisition conditions, the aortic root appears as an hyperintense region. In order to prevent the influence of small hyperintense regions which can appear in some cases, each image $I_{z}(k)$ is normalized between 0 and 1 under the following constraint: High values are clipped until the number of pixels set to 1 is significant (for instance, greater than $0.2 \%$ of the number of pixels in the image $\left.\left|I_{z}(k)\right|\right)$. This normalization is noted $\Xi_{c}$, with $c$ the percentage of pixels set to 1 . Considering that pixels with a value higher than $30 \%$ of the maximum intensity after the normalization are bright pixels, we also introduced $T_{c}$, the binary image that indicates which pixels of the normalization are bright.

For each image frame, bright pixels inside the body (Fig. 5a) are consequently given by:

$A 1_{z}(k)=T_{c}\left(I_{z}(k) \circ A_{z}\right)$

where $\circ$ depicts the Hadamard product of two matrices. According to the first general property of the SV (enumerated in Section 2.2.1), SV are mostly composed of bright pixels. In order to verify the second and the third properties, the images $A 1_{z}(k)$ are then regularized. Holes are filled by geodesic reconstruction, elements which are close to each other are merged by closing, small elements are erased by opening:

$A 2_{z}(k)=\gamma\left(\varphi\left(H\left(A 1_{z}(k)\right), B_{R 3}\right), B_{R 4}\right)$

with $B_{R 3}$ and $B_{R 4}$ two disks coherent with the common sizes of the SV. In order to keep exact shapes, $A 2_{z}(k)$ is then geodesically reconstructed inside $A 1_{z}(k)$ and a dilation by a small structuring element $B_{R 5}$ guarantees that the SV are entirely inside this new set (Fig. 5b):

$$
A 3_{z}(k)=\gamma^{R E C}\left(A 1_{z}(k), A 2_{z}(k) \circ A 1_{z}\right) \oplus B_{R 5}
$$

Finally, considering the fourth and the fifth properties of the $\mathrm{SV}$, a constraint of temporal coherence can be applied. Let $B_{R 6}$ be a small structuring element which simulates acceptable movement and growing. Each bright region of the current frame must be included inside the dilated bright region of the previous and the next frame:

$$
A 4_{z}(k)=\left(A 3_{z}(k-1) \oplus B_{R 6}\right) \circ A 3_{z}(k) \circ\left(A 3_{z}(k+1) \oplus B_{R 6}\right)
$$

For the first (respectively the last) frame, only the existing next (respectively previous) frame is considered. This final set representing bright regions including the SV or the aortic root (Fig. 5c). 


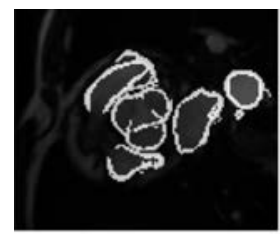

a)

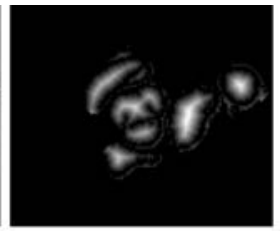

b)

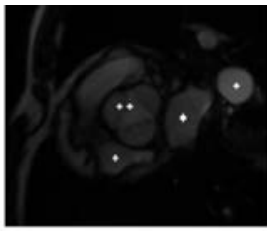

c)

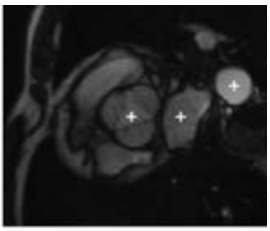

d)

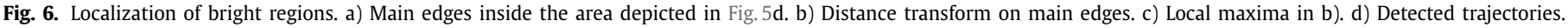
The bottom left point in c) is eliminated. This point is not inside any trajectory since it is missing in most frames.

\subsubsection{Trajectories}

Even if each organ is not separately identified in $A 4_{z}(k)$, a point per region can be estimated in order to track each region. SV and aortic root are star domains whose associated detected points will be near to their center.

Firstly main edges are detected inside the bright regions using the centered differences approximating gradient $\nabla\left(\Xi_{c}(I)\right)$ :

$A 5_{z}(k)=T_{c}\left(\left|\nabla\left(\Xi_{c}\left(I_{z}(k)\right)\right)\right| \circ A 4_{z}(k)\right)$

In order to reduce the artifact of noise and blood flow that can induce isolated irrelevant small edges, edges are eroded by a unit disk and geodesically reconstructed (Fig. 6a):

$$
A 6_{z}(k)=\gamma^{R E C}\left(A 5_{z}(k) \ominus B_{1}, A 5_{z}(k)\right)
$$

In order to find the points that are locally the farthest from the edges inside the bright regions, the Euclidean distance transform $\delta$ [20] is then computed (Fig. 6b):

$$
\left.A 7_{z}(k)=\delta\left(A 6_{z}(k)\right) \circ A 4_{z}(k)\right)
$$

Farthest points are those having a local maximum intensity inside $A 7_{z}(k)$ (Fig. 6c). From these maxima, a trajectory per region is defined. The trajectory of a region is a set composed of one point located inside the region per frame. Closest points from a frame to the next one are aggregated inside the same trajectory (Fig. 6d). Then, abnormal trajectories (containing aberrant or missing points) are filled by barycenters of the two temporally closest points. Considering the distance between each point and the barycenter of the trajectory, some aberrant points are detected using the usual centered confidence interval of six standard deviations.

If the distance between trajectories is less than $30 \mathrm{~mm}$ [21], only one is retained. Natural and not noisy trajectories i.e. less corrected ones are favored. If not, the most stable one is retained using the standard deviation of distances between points and the barycenter. If 3D spatial information is available, trajectories relevancy is increased by selecting similar trajectories over distinct 2D planes. If there are more than one trajectory at the end of the automatic process, the only one which is inside the SV is manually selected. A square Region Of Interest (ROI) centered on the selected trajectory allows to track it. The ROI size is automatically set to $80 \mathrm{~mm}$ [22] in order to completely include the aortic root and the SV on each frame.

\subsection{Polar geodesic reconstruction and aurora transform}

A new approach, based on a polar geodesic reconstruction and a specific transform, is proposed. The extraction of star domains is then simplified, particularly in case of connected organ edges.

\subsubsection{Theory}

The grayscale geodesic reconstruction allows one to recovers an area from a marker $[23,24]$. The reconstruction $\gamma^{R E C}$ of function $f$ from $g$ is the supremum of the geodesic dilations of $g$ inside $f$ :

$\gamma^{R E C}(f, g)=\operatorname{Sup}\left\{\operatorname{Inf}\left(g \oplus B_{r}, f\right), r>0\right\}$
On a binary image $I$, holes of shapes can be filled, whatever their sizes, by considering the geodesic reconstruction of the image border inside the set of black pixels. This operation is noted $H(I)$.

Let $I$ be a square grayscale image of size $2 s+1$ that contains a simply connected shape $I_{0}$ surrounding $p_{0}$, the center of $I$. Let $\widetilde{I}$ be the set of pixels on the border of $I$. Since $I$ is a square, each border pixel is at the same distance from the center considering the Chebyshev distance $\left(\forall p \in \widetilde{I},\left|p-p_{0}\right|_{\infty}=s+1\right)$.

In this discrete set, $I_{0}$ is called a star domain according to $p_{0}$ if all line segments of [25] from $p_{0}$ to each pixel of the edge of $I_{0}$ are completely inside $I_{0}$. This means that in centered polar coordinates, the edge function of the shape is well-defined and can be continuously described (in terms of pixel connectivity). Using this postulate, a new transform and a new reconstruction based on geodesic reconstruction are proposed in order to more easily and more precisely detect the edge of centered star domains inside grayscale images.

The classical geodesic dilations and reconstruction are isotropic. Hence intensities spread in all the directions (Fig. 7b). To correct this point, a spread that is only radial is built, from $p_{0}$ to each pixel of $\widetilde{I}$. For this purpose, Bresenham's line algorithm extracts segments in each direction. This constitutes the linear sets $L(p)$ of radii linked to each pixel $p$ of the border $\widetilde{I}$ to the center $p_{0}$. It does not avoid redundancy but ensures that all pixels of $I$ are used at least once. It also does not exactly provide a constant angular resolution but it is very simple and avoids approximation and interpolation. Moreover, each radius has the same size: $\forall p \in \widetilde{I},|L(p)|=s+1$.

A geodesic reconstruction is separately performed on each set $L(p)$ from the shared core $p_{0}$. All results are then juxtaposed to constitute a two-dimensional matrix where the abscissa corresponds to the direction and the ordinate corresponds to the radius (this is almost a $(r, \theta)$ plane as found in the transform proposed by Radon and Parks [26]). On images containing a centered star domain and surrounding shapes, this polar transform seems like an aurora borealis (Fig. 7d). Hence, we called it the Aurora Transform (AT) and is noted $\Gamma(I)$ where $I$ is an image.

To complete this transform, a Cartesian representation of AT is done and is named the polar geodesic reconstruction as opposed to the classic geodesic reconstruction (Fig. 7c). We see that the spread of intensities is restricted to beam directions and therefore is more limited than with the classic geodesic reconstruction (Fig. 7b).

\subsubsection{Edge extraction}

A first approach, proposed by Blanchard et al. [1] allows a reliable measurement of the SV using cine-MRI, especially for the diagnosis of SV dilations [13]. However when same density organs are connected to SV, some SV edges cannot be correctly found. In order to enhance the quality of the detection and work on static examinations, a new morphological method called Aurora Edge Extractor (AEE) was elaborated and is presented here. Using the vertical component of the centered gradient of the Aurora Transform (Fig. 8c), SV edges can be more precisely detected (Fig. 8b) from MRI sequences and also on static views like in CT. In the next sections, we explain how dynamic examinations are registered to be 


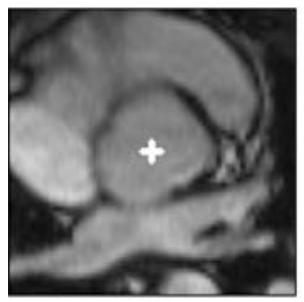

a)

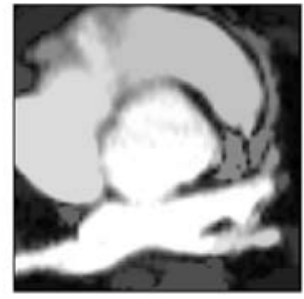

b)

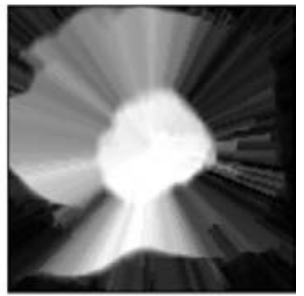

c)

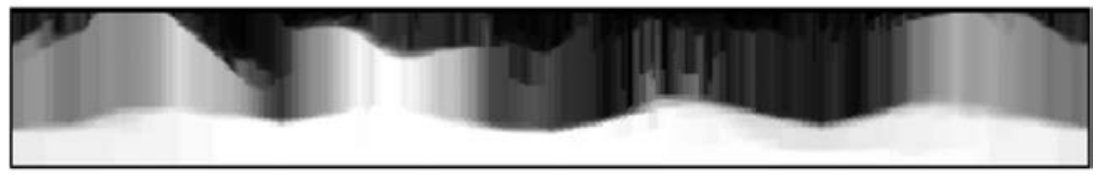

d)

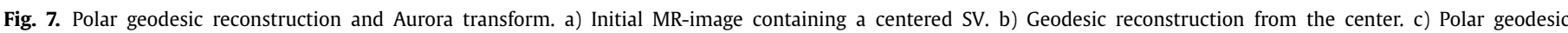
reconstruction. d)Aurora transform $\Gamma(I)$.

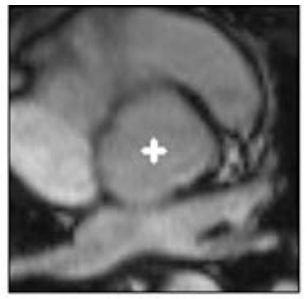

a)

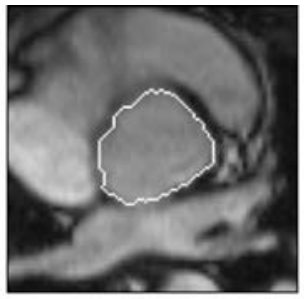

b)

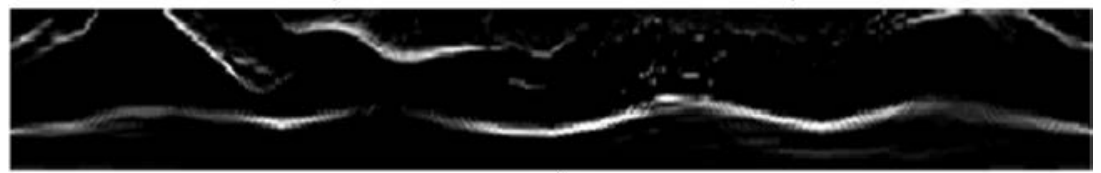

c)

Fig. 8. Edge extraction using the Aurora transform. a) Initial image $I_{k}$ centered on a SV. b) Continuous edge found. c) Vertical gradient of the Aurora transform $\nabla_{\Gamma}^{y}\left(I_{k}\right)$.

in the same place from one image to another and how the radial edge of the SV is extracted.

Considering a single image $I_{k}$ from a sequence $I_{1}, \ldots, I_{n}$ of ROI centered on the SV, let $\nabla_{\Gamma}^{y}\left(I_{k}\right)$ be the vertical component of the centered gradient of the Aurora transform. Main radial edges are sought in $\nabla_{\Gamma}^{y}\left(I_{k}\right)$. Highest intensities are employed as initial markers. From each marker a complete path (without interruption) is drawn by seeking the highest intensity in the left and in the right around the path under construction. If there is no high intensity anymore, i.e. if the path falls in an homogeneous region, the edge is sought in the polar representation of the initial image. A path ends when there is no longer an edge in its neighborhood in $\nabla_{\Gamma}^{y}\left(I_{k}\right)$. The image containing the drawing of all paths is noted $\varepsilon_{1}\left(I_{k}\right)$. After selection of the more contrasted complete path, the inverse of $\varepsilon_{1}$ transform gives the location of main radial edges in the initial image.

\subsubsection{Image registration in dynamic examinations}

Using the number of edge pixels that match between two images as a criterion to maximize, images of the whole examination can be registered and new ROI $I_{1}^{R}, \ldots, I_{n}^{R}$ are extracted so that the $\mathrm{SV}$ is motionless inside them. The registration is dynamically assessed by building a video from these ROI. Its efficiency is also highlighted by building three images before and after the registration: they respectively contain the minimum, the mean and the maximum intensities in each location on the whole sequence (Fig. 9).

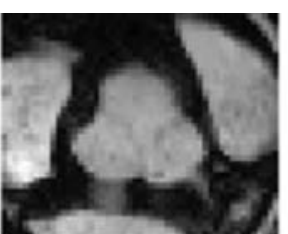

a)

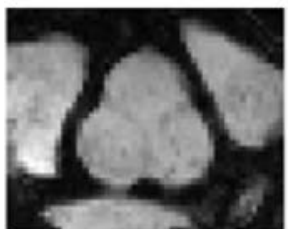

d)

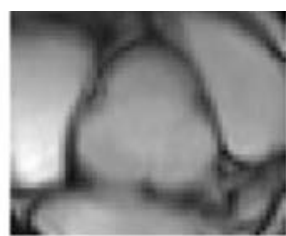

b)

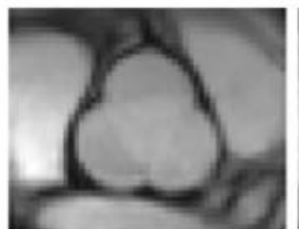

e)

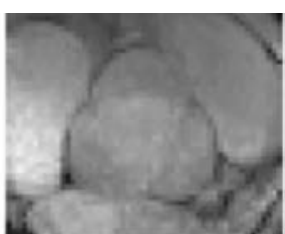

c)

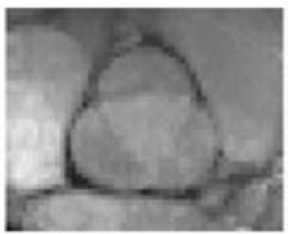

f)
Fig. 9. Visual assessment of the registration. Minimum (a), mean (b) and maximum (c) intensities on a whole sequence before registration. Minimum (d), mean (e) and maximum (f) intensities after registration.

The vertical gradient can then be masked by a region encompassing the main radial edges of the sequence:

$\nabla_{2}^{y}\left(I_{k}^{R}\right)=\nabla_{1}^{y}\left(I_{k}^{R}\right) \circ\left(T_{c}\left(\sum_{n}^{p=1} \varepsilon_{1}\left(I_{p}^{R}\right)\right) \oplus B_{5}\right), \forall k \in[1, n]$ 
Hence vertical gradients that are not relevant are set to zero and do not influence the detection of SV edge.

\subsubsection{Main cyclic radial edge}

The SV contour contained in an image is defined as the inverse transform of the main cyclic path found in the Aurora transform (using $\nabla_{1}^{y}\left(I_{k}\right)$ or $\nabla_{2}^{y}\left(I_{k}^{R}\right)$ if there is registration).

Main radial edges are determined in the same way as for the previous step, drawing paths from highest intensities in $\nabla_{1}^{y}\left(I_{k}\right)$. Then a cyclic path is built by selecting best candidates among all paths found and completed if necessary:

- For each abscissa in $\nabla_{1}^{y}\left(I_{k}\right)$, if one and only one pixel belongs to a path, this position is retained as the edge of the SV for this abscissa.

- Otherwise, if there is an interval of abscissae having none or more than one path, the two extremities of SV edges already found around the interval are linked by the best geodesic path that can be found.

\subsubsection{Spatial and temporal corrections}

Finally each detection is corrected according to temporal (respectively spatial) stability according to the fourth (respectively the sixth) property in Section 2.2.1.

Indeed, in image sequences, the shape drawn by the SV is almost constant. Hence aberrant radii are identified by computing, on the whole cardiac cycle, the mean and the standard deviation of all the radii for each direction. They are then replaced by the barycenter of the two closest reliable radii.

In multi-slice examinations, the difference of the SV shape between two successive slice is very limited. Hence the edge of the SV shape $S_{z}$ at a given level must be included in the neighborhood of the SV edge of neighbor levels:

$\nabla^{\ominus}\left(S_{z}\right) \subset\left(\left(S_{z-1} \oplus B_{R} \cap S_{z+1} \oplus B_{R}\right)-\left(S_{z-1} \ominus B_{R} \cup S_{z+1} \ominus B_{R}\right)\right)$

SV shapes are corrected according to this constraint in CT examinations and multiplane cine-MRI.

\subsection{Extraction of shape and relevant points}

In order to reduce the effect of digitization on the study of the SV shape, the resolution of SV shapes is firstly multiplied by five without interpolation. There is no spatial shift during the increasing of the resolution. To compensate for the bias introduced by the pixel spacing in the initial resolution, an erosion is applied. Thus the final SV shape $S_{Z}^{*}(k)$ is given by the following equation:

$S_{z}^{*}(k)=\left(\varphi\left(\gamma\left(\chi\left(S_{z}(k), 5\right), B_{5}\right), B_{5}\right)\right) \ominus B_{5}$

with $\chi(I, m)$, the nearest-neighbor interpolation of $I$ that increases the resolution by a factor $m$. The relevant points extraction is based on the geometrical propriety of this shape.

\subsubsection{Center of the SV}

Considering the positions of maxima in the Euclidean distance transform of $S_{z}^{*}(k)$, the SV center is the isobarycenter of these locations. This is the same principle as in the automatic localization of the SV with the exception that the edge of the aortic root is now well-defined.

\subsubsection{Local extrema in polar edge study}

Firstly, local extreme radii are sought along the edge of each SV shape. Cusp point candidates are edge pixels where the radius is maximum whereas commissures are edge pixels where the radius is minimum. Since each cusp has only one cusp point and is delimited by two commissures, two points of a same type cannot be close to each other. The radius $R_{e} q$ of a disk of same area as the SV region defines the minimum distance between two points of the same type. This constraint and the temporal or spatial coherence allow to reliably locate commissures and cusp points.

\subsection{Measurement section}

Today, the maximum diameter in SV is employed to evaluate the SV dilations. Nevertheless, some other measurements could be useful. According to the mean number of commissures, SV can be morphologically classified as a bicuspid valve or a tricuspid valve. This classification is not always similar to the functional classification of SV [27], since the study of the SV does not include the detection of raphe between valves. With a knowledge of the position of SV center, commissures and cusps points (Fig.2), our method can provide the following measurements:

- distance between two commissures;

- distance between a cusp and the opposite commissure (for tricuspid);

- distance between a cusp and the two commissures for bicuspid cases;

- distance between two cusps;

- distance between the center and a cusp.

Our method can be successfully used to detect SV dilation [13]. These measures based on relevant points could have more morphological meaning than a maximum arbitrary radius in our mind. The distance between a cusp and the center of the SV could also be a good general measure for SV dilation diagnosis, whatever the morphology of the SV, especially when the dilation is located in only one cusp.

\subsubsection{Detection of the phase of the cardiac cycle}

Using the position of commissures and center in each image, the binary predominance of being a diastolic or a systolic image can be estimated for tricuspid cases.

A Region Of Interest (ROI) for this classification is defined as a geodesic dilation of the commissure filled triangle (Fig. 10). Thus, the ROI completely encompasses the area of valve opening. Afterwards, this ROI is segmented twice in order to define positive and negative regions for diastolic and systolic phases (final regions in Fig. 10).

The systolic positive region $S^{+}$is defined as a geodesic dilation of the segments that connect commissures inside the ROI whereas the diastolic positive region $D^{+}$is built by performing a geodesic dilation of the segments between commissures and the center. The respective negative regions $\left(S^{-}\right.$and $D^{-}$) are simply the relative complement of positive regions with respect to the ROI.

The arithmetic mean $m(R, I)$ that provides the average intensity value of a region $R$ inside an image $I$ allows to build a diastole criterion $T_{\Delta}$ and a systole criterion $T_{\Sigma}$. We propose to compute them using the following formulae:

$T_{\Delta}(I)=m\left(D^{+}, I\right)-\frac{m\left(D^{-}, I\right)}{2}$

$T_{\Sigma}(I)=m\left(S^{+}, I\right)-\frac{m\left(S^{-}, I\right)}{2}$

The mean of the negative region is always divided by two in order to promote the real contours of the valves instead of potential noise induced by blood flow. The maximum of the two criteria gives the phase of the studied image.

\subsubsection{Valve post-process for assisted visualization}

A valve tracking could be useful for the medical practitioner. For example, the correct valves closing and opening can be detected. 


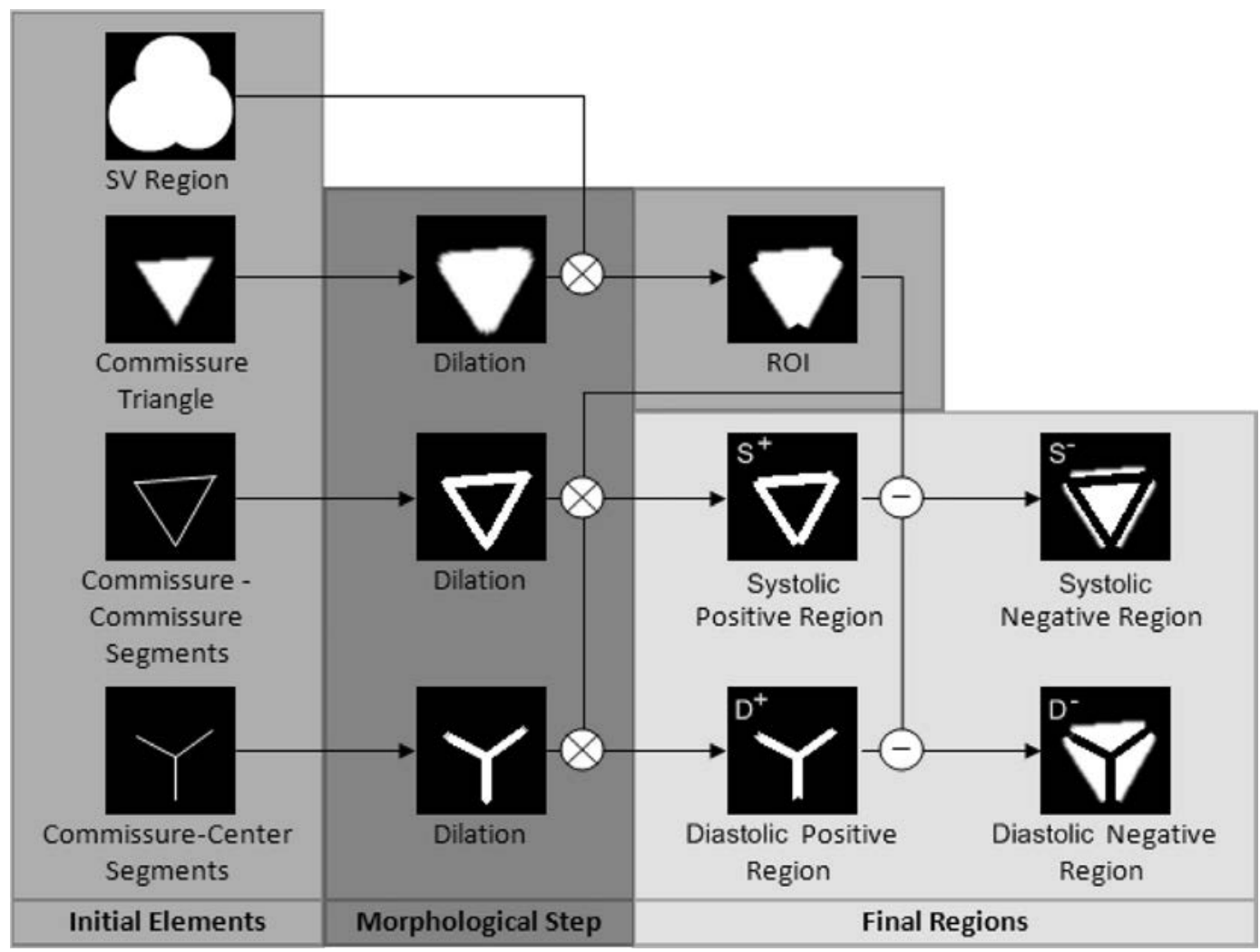

Fig. 10. Diagram of morphological steps used to build diastolic and systolic positive and negative regions.
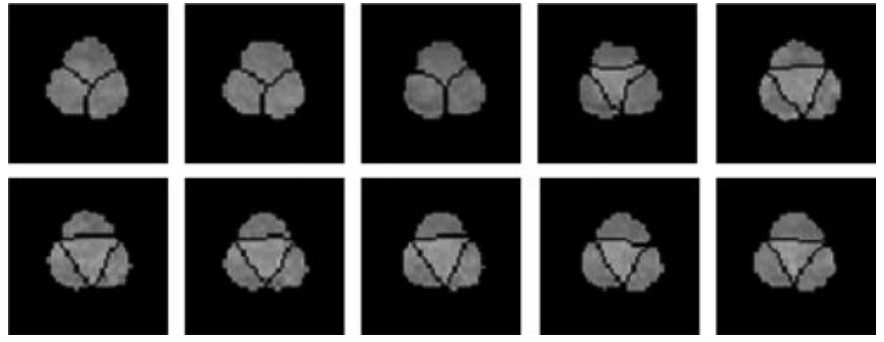

Fig. 11. Detection of valves boundaries using opened snakes. The ten images are from the same cine-MRI examination.

Following the central SV region evolution during the cardiac cycle, a mutual valves edge during systole (respectively a low maximum open area) could indicate a raphe (respectively an aortic stenosis). So precise edges of valves are needed. To find them, an open active contours is used (snakes as presented by Amini et al. [28]). The initialization is done by considering segments between commissures (respectively segments that link commissures and the center) for images of a systolic phase (respectively a diastolic phase). When the minimization ends, each snake is close to valve boundaries due to the grayscale homogeneity in each valve (Fig. 11).

\section{Results}

The experiments were done in Matlab on a dual core $2.4 \mathrm{GHz}$ and 3Go RAM PC. The presentation of results focuses on two aspects. The first section deals with the accuracy of the methods and the second one is centered on processing time considerations.

The statistical values of the processing time, the number of organs found, the number of points localized inside the SV are provided. Moreover, we also explain in which conditions our method
Table 2

Numerical results of the automatic localization of bright organs in our whole data set. For each type of data, the number of success, the mean and the standard deviation (SD) are given.

\begin{tabular}{llll}
\hline & Success $(\mathrm{Y} / \mathrm{N})$ & Mean & $\mathrm{SD}$ \\
\hline Cine-MRI (one plane) & $42 / 2$ & 0.95 & 0.21 \\
Multi-planes cine-MRI & $7 / 1$ & 0.88 & 0.35 \\
CT & $4 / 0$ & 1.00 & 0.00 \\
\hline
\end{tabular}

fails and highlight the difficult cases for which our method was successful.

\subsection{Accuracy}

The automatic localization is processed on all patient examinations. For each examination, the result is a set of seeds located in bright organs on each frame. As the process defines seed trajectories, all seeds are present in each image of the sequence. In case of several trajectories i.e. several bright organs detected (Fig. 12the medical practitioner selects the SV seed by clicking on the correct seed. So, to consider a successful SV localization, only one seed must be located in the SV. That is to say that if there is no seed or more than one seed in the SV, the process is a failure (Fig. 6d) and a manual localization must be done.

The robustness of the localization is assessed by testing it on some difficult cases. Except for three sequences, the localization is always correct, especially with CT exams and even with those that are saved as JPEG images containing embedded text around the SV.

The success rate is equal to $95 \%$ for single plane cine-MRI, $88 \%$ for multiplanes cine-MRI and $100 \%$ for CT. Numerical results of the automatic localization of the SV are given in Table 2. 


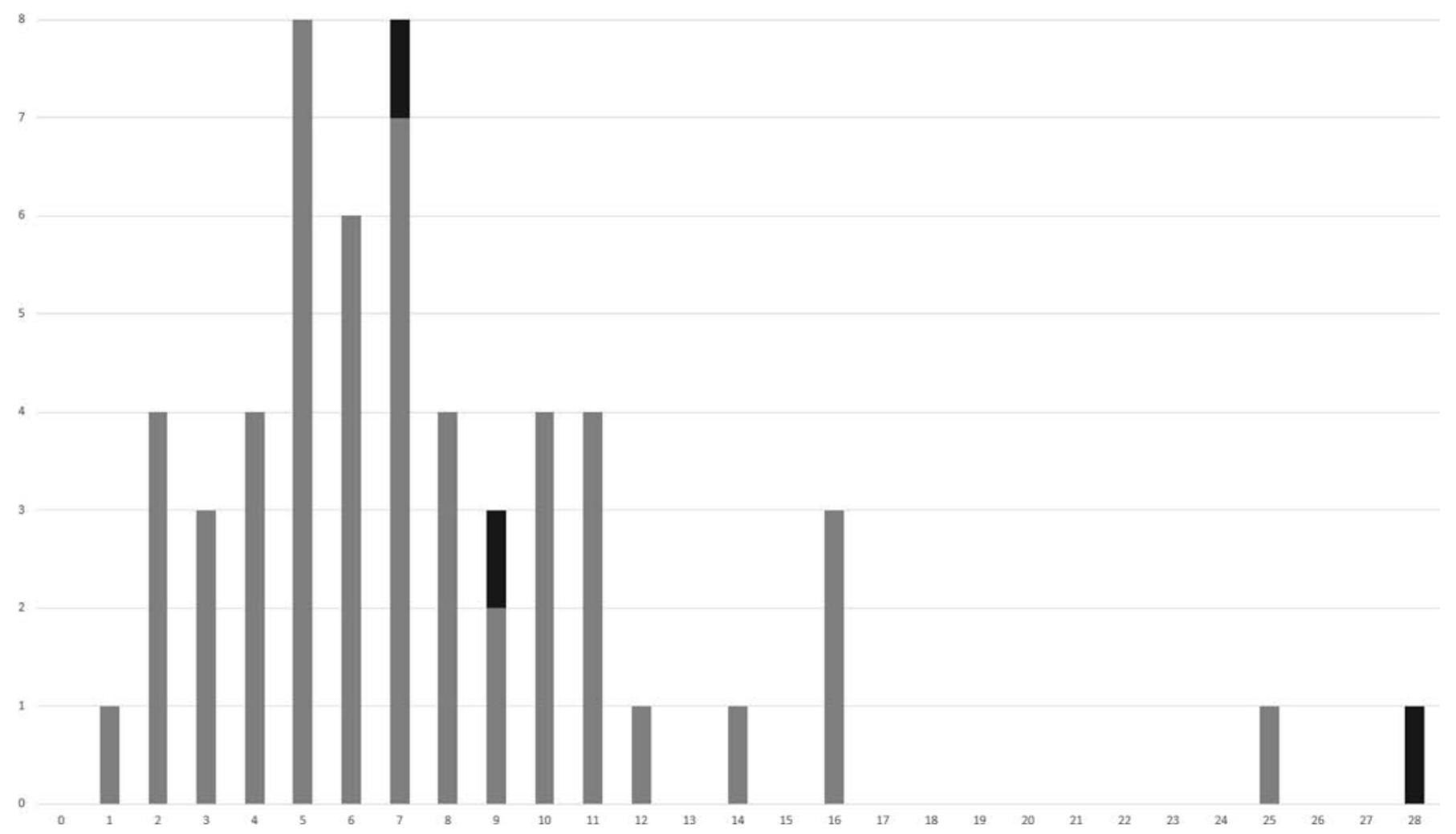

Fig. 12. Frequency histogram of seeds number. Gray color indicates a success and black color a failure.

The edge extraction is the most important step of the study. The results of AEE are compared with the Binarization of the Geodesic Reconstruction (BGR) [1] and the three state-of-the-art methods based on active contours: the Poisson Inverse Gradient (PIG) [29], an active contour by Reaction-Diffusion Network (RDN) [30] and the Region Scalable Fitting (RSF) [31]. In order to be efficient, these methods needs to be parametrized. So, correct parameters have been empirically chosen for each method on the whole data set. The PIG method is a Gradient Vector Flow (GVF) [32] whose external force is the Vector Field Convolution (VFC) [33]. The Poisson Inverse Gradient is used to initialize the VFC. The RDN method is based on the propagation of topological waves. It provides a grayscale picture where each gray level corresponds to a different contour which represents the object viewed at a different scale. This image is then thresholded on a constant scale to obtain the desired contour. The RSF method proposes a region-based active contour. For each side of the contour, a function is defined to fit image intensity. The energy of each region depends on the local difference between the image and the fitting functions. After adding a common smoothing condition by penalizing the length of the contour, the energy function is minimized by a standard gradient descent on a level set formulation making the evolution of the contour. This active contour is able to cope with intensity inhomogeneity which is often present in medical imaging.

Each frame is processed by the five detectors methods and gives five edges to evaluate. The resulting 6200 edges are then randomly mixed in order to perform a blind evaluation by three experts. Separately, the experts assessed each edge in a binary manner. An edge is considered as correct if the maximum distance between it and the expert true edge does not exceed two pixels. From these 18,600 results, an success rate, defined as the ratio between the
Table 3

Comparison between the percentages of success of edge extractor based on geodesic reconstruction and recent active contours (expressed in percent according to the number of images in each examination). Since BGR needs temporal information to be computed, this method is not used on CT examinations.

\begin{tabular}{llllll}
\hline & & Min (Frequency) & Max & Mean & SD \\
\hline \multirow{4}{*}{ Cine-MRI } & PIG & $7(1)$ & 100 & 54 & 27 \\
& RDN & $0(7)$ & 94 & 39 & 26 \\
& RSF & $0(5)$ & 100 & 57 & 27 \\
& BGR & $0(4)$ & 100 & 77 & 26 \\
& AEE & $0(4)$ & 100 & 84 & 24 \\
CT & PIG & $55(1)$ & 86 & 65 & 12 \\
& RDN & $0(5)$ & 77 & 23 & 29 \\
& RSF & $7(1)$ & 100 & 57 & 30 \\
& BGR & - & - & - & - \\
& AEE & $57(1)$ & 100 & 89 & 11 \\
\hline
\end{tabular}

number of correct edges and the number of examined ones, is deduced for each detector, examination and expert. For each detector, the minimum, the maximum, the mean and the standard deviation (SD) of the success rate by examination and expert are given in Table 3. An average success rate by detector and examination over the three experts is given as a boxplot in Fig. 13. An histogram of success rate distribution on MRI data is given in Fig. 14 .

For instance on cine-MRI sequences, the active contours average success rates are between $39 \%$ and $57 \%$. These methods are far from successful every time. For the developed methods, the BGR succeeds in $77 \%$ of images on average. The use of the AEE based on AT further increases the efficiency and the robustness of the SV edge extraction $(84 \% \pm 24 \%)$. The AEE is also the best method on CT examinations $(89 \% \pm 11 \%)$ (Table 3$)$. 


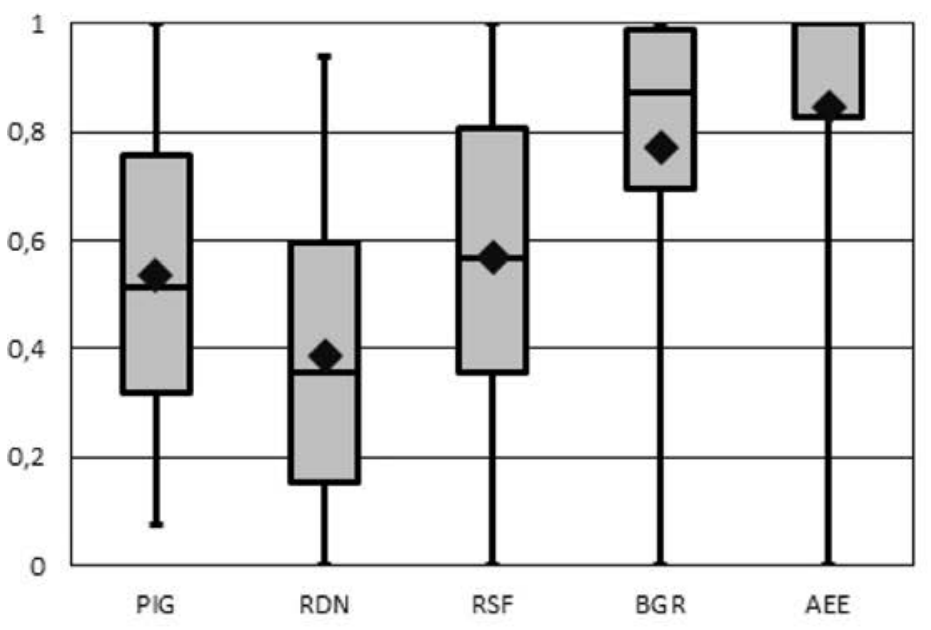

a)

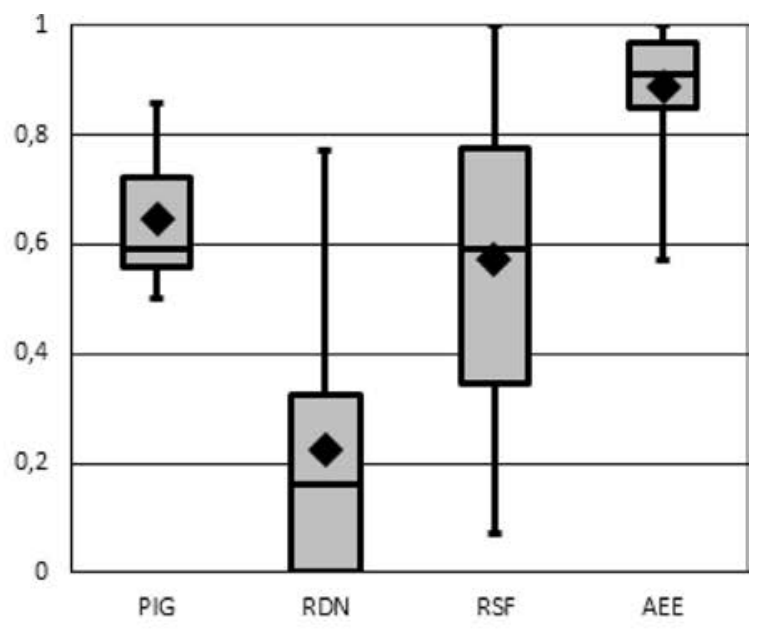

b)

Fig. 13. Graphical comparison between the percentages of success of edge extractors on IRM (a) and CT (b).

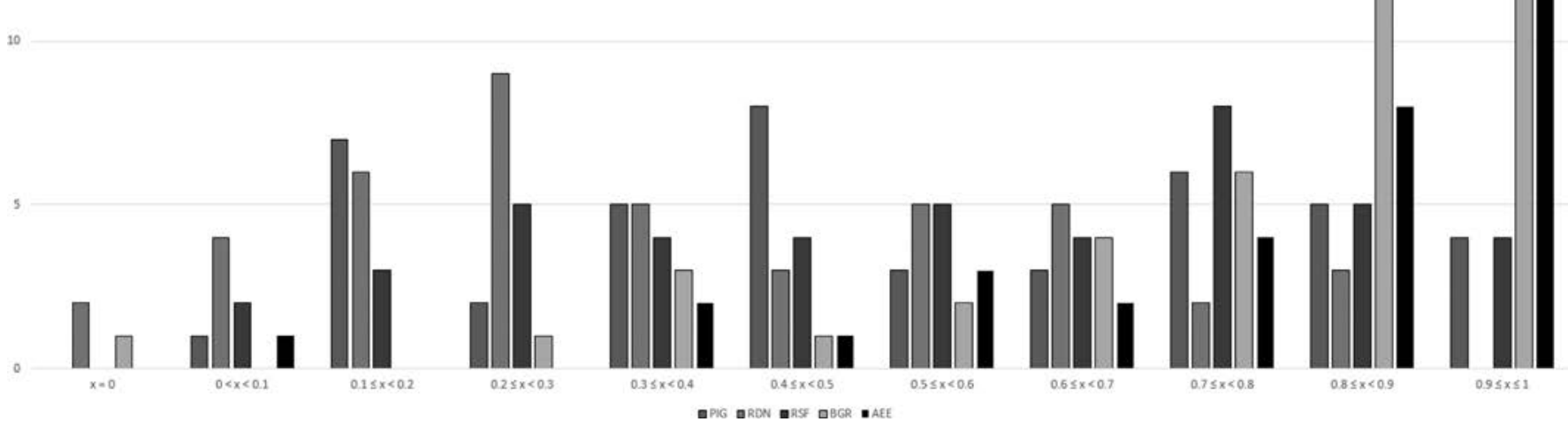

Fig. 14. Frequency histogram of edge extractor methods by mean success rate on MRI data.

Considering the relevant points extraction and measurement, results were obtained using the most successful edge extractor: AEE method. To compare with previous work [1], results with the BGR extractor are shown. Moreover, these methods are automatic and do not need any parametrization for each new examination. Even if active contours are often used to segment medical images, their limits are obvious when the parameters of the segmentation must not be changed from one examination to another or from one image to another. Some examples of AEE results from CT examinations can be shown on Fig. 15. The comparison is performed with a linear regression analysis and the method proposed by Bland and Altman [34] (Fig. 16).

\subsection{Processing time considerations}

The localization processing time is between $7 \mathrm{~s}$ and $2 \mathrm{~min}$ according to the resolution, the size and the number of images in the examination. This time is acceptable to a medical practitioner for a study of a patient examination. Moreover, the processing time can be easily reduced with another implementation (in C language for instance) on a more powerful computer. The statistical values of processing time are given in Table 4.

The edge extraction processing time is shown in Table 5. RDN method is the most time-consuming edge extractor. As the underlying FitzHugh-Nagumo equation is related to physic propagation, it needs a specific electronic implementation [30] to be 


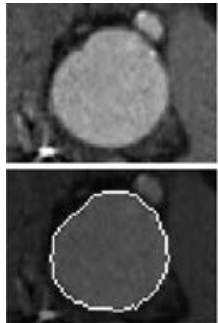

a)

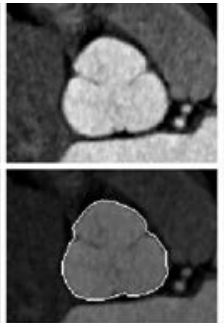

b)

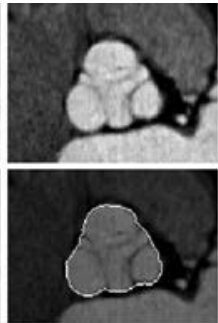

c)

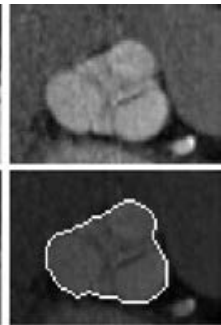

d)
Fig. 15. Some results of AEE from the CT examinations at different levels of the aortic root. Initial ROI are shown on the top and detected edges are drawn at the bottom. (a) Beginning of the ascending aorta (b) Aortic valve cross-section plane (c) and (d) Low levels of the aortic root, under the aortic valve cross-section plane.

Table 4

Computation time (in seconds) of the automatic localization of bright organs in our whole data set. The minimum, the maximum, the mean and the standard deviation (SD) of these values are given.

\begin{tabular}{lllll}
\hline & Min & Max & Mean & SD \\
\hline Cine-MRI (one plane) & 07 & 86 & 42 & 30 \\
Multi-planes cine-MRI & 78 & 124 & 95 & 19 \\
CT & 34 & 70 & 49 & 17 \\
\hline
\end{tabular}

Table 5

Comparison between the speed of edge extractor based on geodesic reconstruction and recent active contours (expressed in second per slice for cine-MRI and in second per examination for $\mathrm{CT}$ ).

\begin{tabular}{llllll}
\hline & & Min & Max & Mean & SD \\
\hline Cine-MRI & PIG & 2.3 & 14.8 & 7.9 & 4.1 \\
& RDN & 25.1 & 3186.7 & 889.7 & 919.7 \\
& RSF & 17.3 & 137.7 & 66.1 & 39.2 \\
& BGR & 0.4 & 27.6 & 5.2 & 5.8 \\
CT & AEE & 12.5 & 96.6 & 45.7 & 27.3 \\
& PIG & 5.5 & 21.2 & 10.0 & 7.5 \\
& RDN & 46.8 & 604.1 & 251.6 & 306.6 \\
& RSF & 46.7 & 200.7 & 91.7 & 73.4 \\
& BGR & - & - & - & - \\
& AEE & 14.2 & 75.9 & 33.0 & 29.0 \\
\hline
\end{tabular}

time-efficient but, as it is, it cannot be used under decent conditions for a medical practitioner. BGR [1] and PIG processing time are close. At most, they take less than $30 \mathrm{~s}$. The average processing time of RSF is close to one minute and the one of AEE is almost equal to $45 \mathrm{~s}$ for cine-MRI sequences. On the data-set, these processing times are acceptable in medical practice.

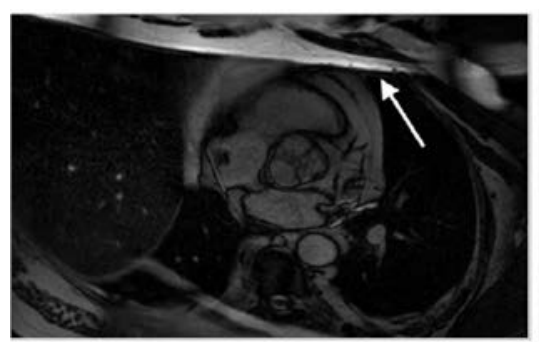

a)

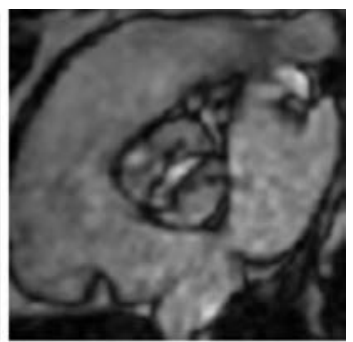

b)
Fig. 17. An artifact and a disease that can affect the correct automatic localization of the SV. a) There is aliasing, mainly at the top of the picture. b) Due to the presence of calcification in an aortic stenosis, valve boundaries are too important and there is a hyperintensity inside the opening of it.

\section{Discussion}

Considering localization results (Table 2 ), the method fails on three over forty four examinations. In the first problematic examination (Fig. 17a), there is an aliasing artifact (a specific artifact in MRI). Even if there is no overlapping near the SV, the proposed normalization of the signal at the beginning of the localization step is not relevant and the process fails. Nevertheless, it has been verified that the issue can be solved by normalizing the image according to the maximum intensity that is not located in the aliasing area. There are some other examinations in the data set with aliasing, but its influence is lower and the localization succeeds without modifying the process. The two other sequences (Fig. 17b) fail due to the presence of calcifications. Indeed, valve boundaries are simultaneously too dark and too thick. Moreover, there is an hyperintensity inside the triangle made by the valve opening which is probably due to important blood flow as a consequence of aortic stenosis. There is currently no solution for this case since the method considers each cusp as a unique bright organ. However, a manual SV localization by click is possible to continue the process but can imply user-dependent results. As it is, the method detects bright organs. This leads to an interaction from the medical practitioner to select the SV among all seeds. If the integration of a priori information could reduce the number of seeds even a unique SV seed. The use of phase contrast IRM also gives interesting results on aorta automatic localization [35].

The study of the edge extraction results is ambiguous. On the opposite of BGR and AEE methods, the active contours methods need a parametrization and it influences results [36]. An approach is to automatically found it thanks to a model [37] but it cannot manage high variations. So, without an examination dependent parameters, the extracted edges quality can strongly vary. Although the AEE method furnishes better results, it is difficult to conclude on accuracy. However, the AEE method gives, in mean, good results. The critical point of the extraction is to obtain, at least, one
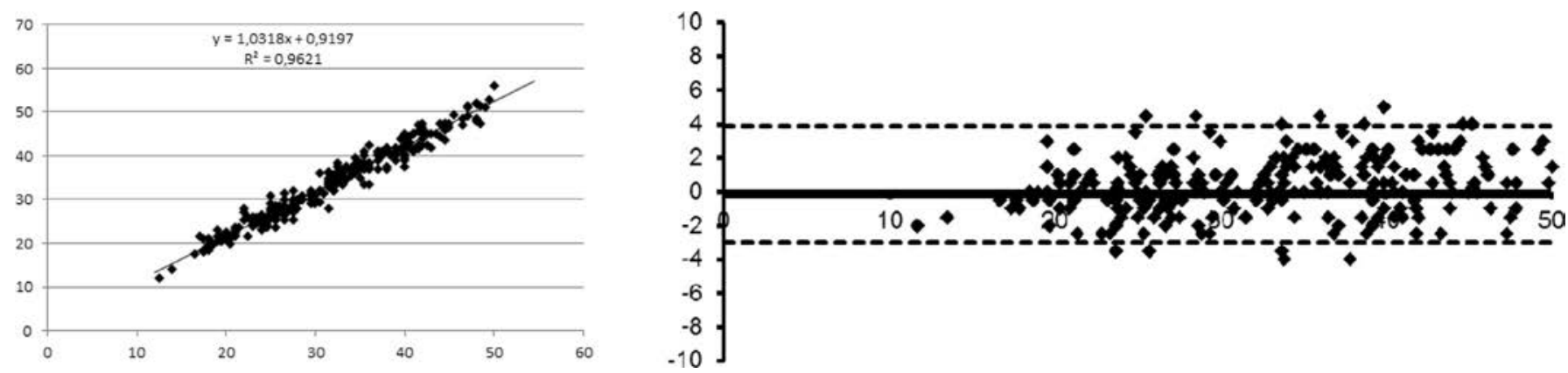

Fig. 16. Linear regression and Bland and Altman analysis results for comparison between automatic and manual measurements. 
Table 6

Comparison of measurement precision deduced from manual locations of relevant points, a detection based on the BGR (need cine-data) and the detection based on the AEE (minimum, maximum, mean and standard deviation (SD) of the differences with this reference in millimeter)

\begin{tabular}{llllll}
\hline & & Min & Max & Mean & SD \\
\hline \multirow{2}{*}{ Cine-MRI } & Inter-observer & -6.0 & 5.2 & 0.7 & 1.9 \\
& BGR & -8.5 & 7.5 & -0.2 & 2.3 \\
& AEE & -5 & 5 & 0.4 & 1.8 \\
CT & Inter-observer & -5.5 & 3 & 0.1 & 1.3 \\
& BGR & - & - & - & - \\
& AEE & -3 & 2 & 0.1 & 1.3 \\
\hline
\end{tabular}

correct edge to get measurements. Considering Table 3, four examinations evaluated by one expert do not return a correct edge with the AEE extractor. In fact, the mean success rate (Fig. 14) is never equal to zero for AEE extractor for the three experts at the same time. Only the RDN and BGR fail, respectively, on two and one examinations. This points at the variability of the experts validation.

Once the relevant points obtained from an edge [1], the measurements can be deduced (Fig. 2). The measurements from the two morphological edge extractors are close to manual measurements (Table 6). Particularly, there is an excellent agreement between the method proposed in this paper and the manual measurement used as the reference for both cine-MRI (difference $=0.4 \pm 1.8 \mathrm{~mm}$ ) and $\mathrm{CT}$ examinations (difference $=0.1 \pm$ $1.3 \mathrm{~mm}$ ). Indeed, there is no bias nor dispersion, whatever the acquisition technique used. On the whole data set, there is a correlation of $r=0.96$ with $y=1.03 x+0.92$.

\section{Conclusions}

In this paper we propose new mathematical morphology tools in order to segment star domains. The Aurora Transform is here applied to automate the study of SV from cine-MRI and static CT examinations. After a description of the main morphological properties of SV, a complete method is proposed in order to automatically localize SV and extract its edge and relevant points. Durations, efficiency and robustness of our process allows to use it in medical practice. The localization is repeatable and robust on the whole data set. The extraction is more efficient than other state-ofthe-art methods. Compared with classical active contours, the AT method enhances the fully automatic edge extraction of star domains such a SV. Moreover, the accuracy of deduced measurements between relevant points are similar to manual measurements. Implemented in a dedicated software, the presented tools will be useful for the follow up of the patients and the surgery of the aortic root. For instance, it will help surgeons to monitor bicuspid SV or diagnose SV dilation and valve diseases. Moreover, the multi-plane extraction of aortic root edges will also eventually allow to design prostheses adapted to each patient in order to improve stress distribution and preserve valves.

\section{Acknowledgment}

Many thanks to Dr Paul M. Walker for his precious help in the redaction of this article, Dr Romaric Loffroy for CT examinations and Pr Olivier Bouchot for his help, his expertise and his medical knowledge. The authors would also like to thank the regional council of Burgundy and the city of Auxerre for supporting this work.

\section{References}

[1] C. Blanchard, A. Lalande, T. Sliwa, O. Bouchot, Y. Voisin, Automatic evaluation of the valsalva sinuses from cine-mri, Magn. Reson. Mater. Phys. Biol. Med. 24 (6) (2011) 359-370.

[2] P. Angelini, Normal and anomalous coronary arteries: definitions and classification, Am. Heart J. 117 (2) (1989) 418-434.

[3] M. Underwood, G. El Khoury, D. Deronck, D. Glineur, R. Dion, The aortic root: structure, function, and surgical reconstruction, Heart 83 (4) (2000) 376

[4] M. Peterson, R. Roach, J. Edwards, Types of aortic stenosis in surgically removed valves, Arch. Pathol. Lab. Med. 109 (9) (1985) 829.

[5] H. Sievert, S. Qureshi, N. Wilson, Z. Hijazi, Interventions in Structural, Valvular and Congenital Heart Disease, CRC Press, 2015.

[6] R. Erbel, V. Aboyans, C. Boileau, E. Bossone, R.D. Bartolomeo, H. Eggebrecht, A. Evangelista, V. Falk, H. Frank, O. Gaemperli, M. Grabenwger, A. Haverich, B. Iung, A.J. Manolis, F. Meijboom, C.A. Nienaber, M. Roffi, H. Rousseau, U. Sechtem, P.A. Sirnes, R.S. von Allmen, C.J. Vrints, 2014 Esc guidelines on the diagnosis and treatment of aortic diseases, Eur. Heart J. 35 (2014) 2873-2926.

[7] H. Patel, G. Deeb, Ascending and arch aorta: pathology, natural history, and treatment, Circulation 118 (2) (2008) 188.

[8] J. Albes, U. Stock, M. Hartrumpf, Restitution of the aortic valve: what is new, what is proven, and what is obsolete? Ann. Thorac. Surg. 80 (4) (2005) 1540-1549.

[9] K. Kunzelman, K. Grande, T. David, R. Cochran, E. Verrier, Aortic root and valve relationships: impact on surgical repair, J. Thorac. Cardiovasc. Surg. 107 (1) (1994) 162.

[10] J.F. Tsang, M. Lytwyn, A. Farag, M. Zeglinski, K. Wallace, M. daSilva, S. Bohonis, J.R. Walker, J.W. Tam, J. Strzelczyk, D.S. Jassal, Multimodality imaging of aortic dimensions: comparison of transthoracic echocardiography with multidetector row computed tomography, Echocardiography 29 (6) (2012) 735-741.

[11] F. Mongeon, F. Marcotte, D. Terrone, Multimodality noninvasive imaging of thoracic aortic aneurysms: time to standardize? Can. J. Cardiol. 32 (1) (2016) 48-59.

[12] M. Roman, R. Devereux, R. Kramer-Fox, J. O'Loughlin, Two-dimensional echocardiographic aortic root dimensions in normal children and adults, Am. J. Cardiol. 64 (8) (1989) 507-512

[13] C. Blanchard, T. Sliwa, A. Lalande, E. Steinmetz, P. Mohan, O. Bouchot, Y. Voisin, Automatic evaluation of the sinus of valsalva from cine-mri in patients with dilated aortic root, J. Cardiovasc. Magn. Reson. 13 (Suppl 1) (2011) 356.

[14] R. Vasan, M. Larson, D. Levy, Determinants of echocardiographic aortic root size: the framingham heart study, Circulation 91 (3) (1995) 734

[15] E. Burman, J. Keegan, P. Kilner, Aortic root measurement by cardiovascular magnetic resonance, Circ.: Cardiovasc. Imaging 1 (2) (2008) 104-113.

[16] J. Rivest, P. Soille, S. Beucher, Morphological gradients, J. Electron Imaging 2 (04) (1993) 326-336

[17] R. Haralick, S. Sternberg, X. Zhuang, Image analysis using mathematical morphology, IEEE Trans. Pattern Anal. Mach. Intell. 9 (4) (1987) 532-550.

[18] J. Serra, Image Analysis and Mathematical Morphology, Academic Press, Inc., Orlando, FL, USA, 1983

[19] P. Soille, Morphological Image Analysis: Principles and Applications, Springer-Verlag, New York, 2003.

[20] C.R. Maurer Jr, R. Qi, V. Raghavan, A linear time algorithm for computing exact euclidean distance transforms of binary images in arbitrary dimensions, IEEE Trans. on Pattern Anal. Mach. Intell. 25 (2) (2003) 265-270.

[21] D. Lavall, H. Schäfers, M. Böhm, U. Laufs, Aneurysms of the ascending aorta, Dtsch Arztebl Int. 109 (13) (2012) 227-233.

[22] L. Pape, T. Tsai, E. Isselbacher, et al., Aortic diameter $5.5 \mathrm{~cm}$ is not a good predictor of type a aortic dissection:observations from the international registry of acute aortic dissection (irad), Circulation (2007) 1120-1127.

[23] P. Salembier, J. Serra, Flat zones filtering, connected operators, and filters by reconstruction, IEEE Trans. Image Process. 4 (8) (1995) 1153-1160.

[24] L. Vincent, Morphological grayscale reconstruction in image analysis: applications and effective algorithms, IEEE Trans. Image Process. 2 (2) (1993) 176-201.

[25] J. Bresenham, Algorithm for computer control of a digital plotter, IBM Syst. J. 4 (1) (1965) 25-30.

[26] J. Radon, P. Parks, On the determination of functions from their integral values along certain manifolds, IEEE Trans. Med. Imaging 5 (4) (1986) 170-176.

27] H. Sievers, C. Schmidtke, A classification system for the bicuspid aortic valve from 304 surgical specimens, J. Thorac. Cardiovasc. Surg. 133 (5) (2007) 1226-1233.

[28] A.A. Amini, T.E. Weymouth, R.C. Jain, Using dynamic programming for solving variational problems in vision, IEEE Trans. on Pattern Anal. Mach. Intell. 12 (9) (1990) 855-867.

[29] B. Li, S. Acton, Automatic active model initialization via poisson inverse gradient, IEEE Trans. Image Process. 17 (8) (2008) 1406-1420.

[30] S. Binczak, T. Sliwa, S. Jacquir, J. Bilbault, Reaction-diffusion network for geometric multiscale high speed image processing, Image Vis. Comput. 28 (6) (2010) 914-926.

[31] C. Li, C. Kao, J. Gore, Z. Ding, Minimization of region-scalable fitting energy for image segmentation, IEEE Trans. Image Process. 17 (10) (2008) 1940-1949.

[32] C. Xu, J. Prince, Snakes, shapes, and gradient vector flow, IEEE Trans. Image Process. 7 (3) (1998) 359-369.

[33] B. Li, S. Acton, Active contour external force using vector field convolution for image segmentation, IEEE Trans. Image Process. 16 (8) (2007) 2096-2106. 
[34] J. Bland, D. Altman, Statistical methods for assessing agreement between two methods of clinical measurement, Lancet 327 (8476) (1986) 307-310.

[35] A. Goel, R. McColl, K. King, A. Whittemore, R. Peshock, Fully automated tool to identify the aorta and compute flow using phase-contrast mri: validation and application in a large population based study., J. Magn. Reson. Imaging 40 (1) (2014) 221-228
[36] S. Pezold, A. Horvth, K. Fundana, C. Tsagkas, M. Andlov, K. Weier, M. Amann, P. Cattin, Automatic, robust, and globally optimal segmentation of tubular structures, MICCAI, 9902, 2016.

[37] J. Wang, C. Shi, Automatic construction of statistical shape models using deformable simplex meshes with vector field convolution energy, Biomed. Eng. Online 16 (49) (2017) 1-19. 